Krisen und Katastrophen in der Fußballberichterstattung aus korpusanalytischer Sicht

Crises et catastrophes dans les commentaires de foot de la presse sportivela perspective de la linguistique de corpus

Crises and Catastrophes in Football Coverage from a Corpus Linguistic

Perspective

\title{
Simon Meier
}

\section{(2) OpenEdition}

\section{Journals}

Édition électronique

URL : http://journals.openedition.org/ceg/2326

DOI : $10.4000 /$ ceg. 2326

ISSN : 2605-8359

\section{Éditeur}

Presses Universitaires de Provence

Édition imprimée

Date de publication : 26 octobre 2017

Pagination : 77-96

ISBN : 979-10-320-0129-5

ISSN : 0751-4239

\section{Référence électronique}

Simon Meier, « Krisen und Katastrophen in der Fußballberichterstattung aus korpusanalytischer Sicht », Cahiers d'Études Germaniques [Online], 73 | 2017, Online erschienen am: 26 April 2019, abgerufen am 24 November 2020. URL : http://journals.openedition.org/ceg/2326 ; DOI : https://doi.org/10.4000/ ceg.2326 


\section{Krisen und Katastrophen in der Fußballberichterstattung aus korpusanalytischer Sicht}

Simon MEIER

Technische Universität Berlin

\section{Einleitung}

Am 18. September 2016 titelt die Berliner Morgenpost: „Gladbach verschärft die Krise von Bremen." Anlass ist die 0:4-Niederlage des SV Werder Bremen vom Vorabend, die die vierte Niederlage im vierten Pflichtspiel der noch jungen Saison bedeutet. Im Artikel wird der Kapitän der Bremer mit folgenden Worten zitiert: „Die erste Hälfte war eine Katastrophe, das war peinlich. Wir können froh sein, dass es nur vier Gegentore waren. Die zweite Hälfte war dann etwas besser" (Berliner Morgenpost, 18.09.2016).

Für routinierte Leserinnen und Leser der Presseberichterstattung über Fußball dürfte dieser Artikel nicht im mindesten außergewöhnlich sein. Krisen und Katastrophen sind im Fußball offenbar allgegenwärtig. Genauer gesagt ist das Reden über Krisen und Katastrophen allgegenwärtig, und mitunter monieren die Protagonisten des Profifußballs selbst, dass in der Presse Krisen "oft herbeigeredet oder -geschrieben" (Bayern-Trainer Jupp Heynckes in Die Zeit, 04.11.2012) ${ }^{1}$ werden. Tatsächlich dürfte ein Charakteristikum von Krisen und Katastrophen im Fußball ihre Schnelllebigkeit sein. Manchmal genügt bereits eine einzige Niederlage eines ansonsten erfolgreichen Teams oder eine zwei Spiele andauernde ,Torflaute' eines sonst erfolgreichen Stürmers, damit in der Presse eine Krise ausgerufen wird. Ein frühes Gegentor kann auch dann als Katastrophe bezeichnet werden, wenn der Rest des Spiels gut gelaufen ist. So gab etwa ein Augsburger Spieler nach dem entscheidenden Sieg für den Aufstieg in die 1. Bundesliga, und somit nach dem größten Erfolg der Vereinsgeschichte, zu Protokoll: „Das Spiel ging mit einer Katastrophe in der zweiten Minute los, danach haben wir 88 Minuten auf ein Tor gespielt, und dass es dann mit dem

1. Dieser und alle weiteren Korpusbelege stammen aus dem für diesen Beitrag definierten (öffentlich zugänglichen) Untersuchungskorpus, das unten (Teil 1) vorgestellt wird. Auf präzisere Quellenangaben der einzelnen Belege wird darum verzichtet, da sie zum Auffinden der Belegstellen nicht nötig sind. 
gefühlten 164. Standard geklappt hat, ist natürlich Wahnsinn" (Süddeutsche Zeitung, 08.05.2011).

Offenkundig ist derartiges Reden über Krisen und Katastrophen Ausdruck eines dramatisierenden Sprachgebrauchs, der für die Fußballberichterstattung insgesamt typisch ist (vgl. Jung 2009; Meier 2016). Akteure der medialen Inszenierung von Fußball, und dazu gehören die Journalisten ebenso wie die Trainer und Spieler, die etwa in Pressekonferenzen und Interviews Auskunft geben, neigen zu Übertreibungen und drastischen Formulierungen, die ihnen die so wichtige Aufmerksamkeit der Fans und Sponsoren sichern. Mit wirtschaftlichen und sozialpolitischen Krisen, wie etwa der Finanz- und Bankenkrise 2008/09, oder mit Technik- und Umweltkatastrophen, wie der Havarie des Atomkraftwerks in Fukushima 2011, haben Krisen und Katastrophen im Fußball wohl nur wenig gemein.

Es wäre aber zu kurz gegriffen, Krisen und Katastrophen im Fußball schlicht als Miniaturausgaben ,echter' Krisen und Katastrophen zu begreifen, die im medialen Diskurs größer gemacht werden, als sie in Wirklichkeit sind. Hier soll vielmehr die These vertreten werden, dass es sich um durchaus eigenständige Konzeptualisierungen handelt, die systematisch auf Besonderheiten der sprachlichen Form bezogen werden können. Die Grundbedeutungen dieser beiden Lexeme, die in Anlehnung an elexiko, einem korpusbasierten Wörterbuch zur deutschen Gegenwartssprache ${ }^{2}$ mit "schwierige Situation“ bzw. „folgenschwerer Unglücksfall" umschrieben werden können, differenzieren sich in diesem thematischen Bereich auf spezifische Weisen kontextuell aus (vgl. Schwarz 2002: 281f.), die sich in wiederkehrenden sprachlichen Mustern an der Sprachoberfläche zeigen. Dies soll hier mit korpusanalytischen Methoden untersucht werden, die computergestützt die Auswertung großer Datenmengen erlauben und insbesondere Musterhaftigkeiten im Sprachgebrauch (vgl. Bubenhofer 2009) zutage fördern können. Diese Musterhaftigkeiten können als sprachliche Sedimentierungen typischer Verwendungssituationen gedeutet werden (vgl. Müller 2012: 36) und werfen somit Licht auf die kommunikativen Routinen im diskursiven Feld des medialen Fußballs.

Ich werde im Folgenden also nach den typischen lexikalisch-syntaktischen Kontexten der Ausdrücke Krise und Katastrophe fragen. Dazu werde ich zunächst die Datenbasis vorstellen und anschließend die korpusanalytischen Befunde, insbesondere zu den Kookkurrenzprofilen der untersuchten Lexeme darlegen. Wie ich zeigen werde, können diese Befunde pragmatisch angereichert werden, indem sie etwa auf typische Akteurskonstellationen im medialen Fußball bezogen werden. Davon ausgehend soll abschließend gefragt werden, was dies über die gesellschaftliche Wertigkeit des medialen Fußballs aussagt.

2. elexiko. Online-Wörterbuch zur deutschen Gegenwartssprache, [http://www.owid.de/wb/elexiko/ start.html], Stand: 12.12.2016. 


\section{Korpus und korpusanalytische Methoden}

Die folgenden Ausführungen stützen sich im Wesentlichen auf Texte der Fußballberichterstattung in der Presse, bei denen es sich typischerweise um Vor- und Nachberichte zu Fußballspielen oder auch Reportagen zu einzelnen Vereinen und Spielerinnen und Spielern handelt. Eine Besonderheit dieser Texte ist der hohe Anteil an Redewiedergaben, was vor allem für den Gebrauch des Ausdrucks Katastrophe relevant ist. In Seitenblicken werde ich mich vereinzelt auch auf Fußball-Liveticker (vgl. Hauser 2010) der ganz auf Fußball spezialisierten Onlinemedien [www.weltfussball.de] und [www.sportal.de] sowie die dort publizierten Spielberichte stützen (Meier 2017).

Die Pressetexte wurden dem öffentlich zugänglichen Deutschen Referenzkorpus (DeReKo) des IDS Mannheim entnommen (Institut für Deutsche Sprache 2016). Das webbasierte Analysetool COSMAS II, mit dem DeReKo durchsucht werden kann, erlaubt die für die Zwecke dieser Untersuchung überaus praktische Sortierung der Belege nach Themen (vgl. Weiß 2005), die u.a. "Sport: Fußball" als Kategorie vorsieht. Auf diese Weise können gezielt Texte bzw. Textausschnitte aus diesem Themenbereich erhoben und für die weitere Analyse heruntergeladen werden. ${ }^{3}$ Das für den vorliegenden Aufsatz definierte Untersuchungskorpus setzt sich dabei aus den dort verfügbaren Ausgaben von insgesamt einundzwanzig deutschsprachigen Tages- und Wochenzeitungen seit Januar 2006 zusammen und umfasst rund 2,83 Milliarden laufende Wörter. ${ }^{4}$ Gesucht wurde nach den Ausdrücken \&Krise und \&Katastrophe (bei Suchen mit dem Wortformoperator $\&$ werden auch alle flektierten Formen miterfasst). Die Suche ergab die folgende Anzahl an Treffern: ${ }^{5}$

\section{\&Krise}

Treffer gesamt $268279(94,9$ pMW)

14794 (47,2 pMW)

\section{\&Katastrophe}

$83825(29,65$ pMW $)$

$4952(15,8$ pMW)

Tabelle 1: Anzahl Belege Zeitungstexte (DeReKo)

Mit COSMAS II können verschiedenste korpusanalytische Auswertungen vorgenommen werden. Da bei vielen Tools wie etwa der Kookkurrenzanalyse eine Eingrenzung auf die Treffer zu bestimmten Themen jedoch nicht möglich ist, wurden die Belege mit dem maximal möglichen Kontext von drei Absätzen davor und danach exportiert. Bei den Treffern zu \&Krise war systembedingt eine

3. Die Annotation nach Themen ist allerdings nicht fehlerfrei; vereinzelt werden z.B. Texte über andere Sportarten dem Thema „Sport: Fußball“ zugewiesen.

4. Die berücksichtigten Zeitungen sind: Braunschweiger Zeitung, Burgenländische Volkszeitung, Die Presse, Die Südostschweiz, die tageszeitung, Die Zeit, Hamburger Morgenpost, Hannoversche Allgemeine, Luxemburger Tageblatt, Mannheimer Morgen, Neue Zürcher Zeitung, Niederösterreichische Nachrichten, Nordkurier, Nürnberger Nachrichten, Nürnberger Zeitung, NZZ am Sonntag, Rhein-Zeitung, Sonntagsblick, St. Galler Tagblatt, Süddeutsche Zeitung.

5. Die Tabelle gibt die absoluten Häufigkeiten und in Klammern die relativen Häufigkeiten pro Millionen Wörter (pMW) an. 
Zufallsauswahl von 10000 notwendig. Die auf diese Weise erstellten Korpora (im Folgenden Fußballkorpora genannt) haben den folgenden Umfang: ${ }^{6}$

\&Krise \&Katastrophe

Laufende Wörter

1486771

822307

Tabelle 2: Umfang Fußballkorpora

Im Folgenden kommen im Wesentlichen drei korpusanalytische Verfahren zum Einsatz, die sich wechselseitig ergänzen: 1) Kookkurrenzanalysen, 2) Konkordanzanalysen und 3) Keywordanalysen.

1) Kookkurrenzanalysen erlauben die statistisch fundierte Erhebung typischer Wortverbindungen in einem Korpus. Computergestützt wird ermittelt, ob in einem vordefinierten Kotext (hier, dem Standard entsprechend, von 5 Wörtern) zu einem Suchausdruck bestimmte Ausdrücke, die sogenannten Kookkurrenzpartner oder Kollokatoren, signifikant häufiger auftreten, als es gemäß einer reinen Zufallsverteilung erwartbar gewesen wäre (vgl. Steyer 2002: 219; Lemnitzer / Zinsmeister 2010: 143f.). Die auf diese Weise ermittelten Listen von Kookkurrenzpartnern, die Kookkurrenzprofile, welche nicht nach absoluter Häufigkeit, sondern nach ihrem statistischen Assoziationsgrad sortiert werden, vermitteln nicht nur ein differenziertes Bild der kontextuell spezifizierten Semantik der untersuchten Lexeme, sondern indizieren, wie unten zu zeigen sein wird, auch typische pragmatische Verwendungskontexte und Sprechereinstellungen (vgl. Steyer 2004). Für die Kookkurrenzanalyse wurde das Ngram Statistics Package 1.29 (Ranerjee / Pedersen 2003) verwendet. Die Software zählt sämtliche Typen von Wortpaaren in einem Korpus mit einem Abstand von bis zu fünf Wörtern aus und sortiert sie nach statistischer Signifikanz. Aus diesen sehr umfangreichen Wortpaarlisten lassen sich in einem zweiten Schritt alle Wortpaare extrahieren, in denen ein Suchausdruck (etwa Krise) vorkommt. Als statistisches Maß wurde Pearson's Chi-Squared Test verwendet.

2) Konkordanzen, auch Keywords in Context (KWIC) genannt, erlauben die übersichtliche Anzeige von Suchausdrücken in ihren kotextuellen Umgebungen, die bei Bedarf nach bestimmten Kriterien sortiert und ausgezählt werden können. Auf diese Weise können die Hinweise, welche die Kookkurrenzanalysen liefern, anhand größerer Kotextausschnitte spezifiziert werden. Für die Konkordanzanalysen wurde die Software AntConc (Anthony 2005) verwendet.

3) Keywordanalysen dienen der Ermittlung von Ausdrücken, die in einem Korpus relativ zu einem Referenzkorpus signifikant häufiger auftreten und deshalb als typisch für das Korpus gelten können (vgl. Bondi 2010). Hier

6. Der Export von Textausschnitten ist nicht unproblematisch, da Texte, in denen der gesuchte Ausdruck mehrfach vorkommt, mehrfach exportiert werden. Eine manuelle Bereinigung um Dubletten wäre äußerst aufwendig. Bei einer derart großen Anzahl von Treffern kann aber davon ausgegangen werden, dass sich die Effekte etwaiger Doppelungen wieder ausgleichen werden. 
werden die themenspezifischen Belegsätze zu Krise und Katastrophe aus den Fußballkorpora mit Belegsätzen aus einem thematisch unspezifischen Korpus von Onlinenachrichtentexten kontrastiert. Auf diese Weise kann überprüft werden, ob es sich bei den anhand der Kookkurrenzanalysen beschriebenen sprachlichen Phänomenen tatsächlich um fußballspezifische Phänomene handelt. Für das Referenzkorpus greife ich auf die Korpusausschnitte zurück, die das Wortschatzportal der Universität Leipzig ${ }^{7}$ zum Download bereitstellt. Es handelt sich um zwei automatisiert erhobene Sets von je 3 Millionen Sätzen aus deutschsprachigen Onlinenachrichtenportalen aus den Jahren 2014 und 2015. Aus diesen Sets wurden sämtliche Sätze extrahiert, die Wortformen von Krise und Katastrophe enthalten. Es ergeben sich die folgenden Belegzahlen:

\begin{tabular}{crr} 
& Krise & Katastrophe \\
\hline Anzahl Sätze & 7296 & 1862 \\
Anzahl Wörter & 151639 & 38000 \\
\hline
\end{tabular}

Tab. 3: Umfang Referenzkorpora

Für die Keywordanalysen wurden ebenfalls die Software AntConc verwendet, das statistische Maß war auch hier Pearson's Chi Square Test.

Die Anwendung quantitativ-korpusanalytischer Methoden erfüllt hier ausdrücklich heuristische Funktionen. Sie sichern zwar Objektivität und Reproduzierbarkeit der Befunde, doch sind diese natürlich gleichwohl interpretationsbedürftig und müssen stets wieder auf das individuelle Vorwissen des Analysierenden bezogen werden. Die Rohdaten, welche die Analysesoftware ausgibt, also im wesentlichen Wörter- bzw. Wortpaarlisten, sind deshalb eher als Input für die Generierung von Hypothesen zu verstehen, die es anschließend in Auseinandersetzung mit den untersuchten Texten zu spezifizieren und zu prüfen gilt. Gegenüber rein hermeneutischen und erst recht introspektiven Zugängen sind korpusanalytische Verfahren aber schon wegen der Möglichkeit der vollständigen Auswertung auch großer Datenmengen eine Alternative.

\section{Empirische Befunde}

\section{Kookkurrenzprofil von Krise}

Besonders signifikante Kookkurrenzpartner von Krise sind unter anderem und nach absteigenden Signifikanzwerten sortiert (flektierte Formen wurden teilweise zusammengefasst, Groß- und Kleinschreibung wurde nicht beachtet): ${ }^{8}$

7. Wortschatz Universität Leipzig, [http://corpora.uni-leipzig.de/de], Stand: 12.12.2016.

8. Die Beschränkung auf 25 Kookkurrenzpartner ist darstellungstechnisch bedingt und statistisch nicht zu begründen. Die Signifikanzwerte $\left(X^{2}\right)$ liegen zwischen 3231 und 91 somit weit über dem kritischen Wert von 10,33 für $p<0,001$. 
aus, tiefer, in, steckt, sportliche, tief, schritt, gestürzt, überwunden, weg, geschossen, sprechen, verschärft, spitzt, rutscht, schwersten, schlittert, bewältigen, einreden, ausweg, wort, befreien, welche, aktuellen, anhaltenden.

Diese Wortliste ist für sich genommen kaum aussagekräftig, vermittelt aber dennoch erste Eindrücke über typische Kotexte. Auffällig sind zunächst die Präpositionen aus und in. In etwa $35 \%$ aller Belege bildet Krise den nominalen Kern der Präpositionalphrasen in der / die Krise und aus der Krise. ${ }^{9}$ Auch die Vielzahl anderer Ausdrücke mit raumbezogener Semantik (tief, gestürzt, weg, rutscht, schlittert, ausweg) fällt ins Auge. Entsprechende Konkordanzanalysen zeigen, dass all diese Ausdrücke oft zu raummetaphorischen Ausdrücken kombiniert werden: steckt tief in der Krise, rutscht / schlittert / stürzt tiefer in die Krise, Schritt / Weg / Ausweg aus der Krise. Charakteristische Beispiele sind etwa diese hier:

(1) Fabian Schmidt hat mit seinem Siegtor zum 3:2 (1:1) kurz vor Schluss seiner SG Neuwied etwas Luft im Abstiegskampf der Fußball-Bezirksliga Ost verschafft und die SG Herdorf noch tiefer in die Krise geschossen. (Rhein-Zeitung, 04.12.06)

(2) Im Tabellenkeller landete 1860 München mit dem 3:0 bei Aufsteiger FSV Frankfurt einen Befreiungsschlag, während der FC Augsburg mit dem 1:2 bei Rot-Weiß Oberhausen nach fünf Niederlagen in Serie immer tiefer in die Krise rutscht. (Nordkurier, 04.10.08)

Man kann diese Redeweisen als sprachliche Manifestationen einer auch in Diskursen über Wirtschaftskrisen nachweisbaren konzeptuellen Metapher beschreiben, die positiv und negativ bewertete Sachverhalte auf einer räumlichvertikalen Achse nach dem Muster "gut ist oben, schlecht ist unten" anordnet (vgl. Wengeler / Ziem 2010: 347-349). Die Kollokation tiefe Krise bzw. tief in der Krise ist entsprechend auch in anderen thematischen Feldern gebräuchlich. Im Bereich des Fußballs dürfte diese Redeweise aber noch zusätzlich motiviert sein durch die in Spielberichten so wichtige "Tabellensprache" (Burkhardt 2006: 65f.), die in ähnlicher Weise raummetaphorisch geprägt ist. Da Fußballergebnisse auch und gerade in Tabellenform präsentiert werden, führen wiederholte Niederlagen zu einem Abwärtstrend oder einer Talfahrt in der Tabelle, möglicherweise bis in den Tabellenkeller (vgl. hierzu auch Küster 2010) - alle diese Metaphern sind auch im Korpus im unmittelbaren Umfeld von Krise belegt:

(3) Duisburger Talfahrt: Der VfL Bochum stürzt den MSV mit dem 2:0-Auswärtssieg noch tiefer in die Krise. (Hannoversche Allgemeine, 10.11.07)

(4) Die Dresdner bleiben nach der vierten Niederlage hintereinander in der Krise und im Tabellenkeller stecken. (Braunschweiger Zeitung, 24.11.08)

(5) Kevin Kuranyi hat sich aus der schwersten Krise seiner Karriere geschossen und zugleich den Abwärtstrend seines Klubs Schalke 04 gestoppt. (Nürnberger Zeitung, 24.10.08)

9. Noch eindeutiger fällt dieser Befund bei einem Korpus von 1175 Spielberichten zu Bundesligapartien von der Internetseite [www.sportal.de] aus. Von den insgesamt 90 Belegen von Krise entfallen 65 (72 \%) auf die Nominalphrasen in / aus die / der Krise. In 3060 BundesligaLivetickern von [www.weltfussball.de] sind es 161 von 286 (56\%). 
Betrachtet man diese raummetaphorischen Konzeptualisierungen genauer, so fällt zudem auf, dass insbesondere die Dynamik von Krisen thematisiert wird. Nicht allein die Tatsache, dass sich ein Verein in einer schwierigen Lage befindet und in einer Krise steckt, gibt den Meldungen Anlass, sondern mehr noch, dass er in sie hinein- oder aus ihr herausgerät, dass sich eine Krise also verschärft und zuspitzt oder aber überwunden und gemeistert wird. Im Ligabetrieb mit zumeist wöchentlich stattfindenden Spielen können Krisen rasch einsetzen und ebenso rasch wieder enden. Aufschlussreich ist in diesem Zusammenhang die in Beispiel 5 und im Korpus noch weitere 150 mal belegte Wendung sich aus der Krise schießen, mit der meist einzelne Siege nach einer Reihe von Niederlagen bezeichnet werden:

(6) Die "Wölfe" haben sich mit einem klaren Sieg zum Champions-League-Auftakt gegen Moskau und drei Auswärtspunkten in Schalke wieder aus der Krise geschossen. (Braunschweiger Zeitung, 23.09.09)

(7) Chelsea schiesst sich nach dem Trainerwechsel von Mourinho zu Grant mit einem 2:1 in Valencia aus der Krise. (St. Galler Tagblatt, 04.10.07)

Angesichts dieser Schnelllebigkeit von Krisen im Fußball überrascht auch nicht, dass die im themenunspezifischen Kookkurrenzprofil aller 268279 Belege von COSMAS II als signifikant errechneten Formulierungsmuster in Zeiten der Krise oder auch Auswirkungen der Krise und von der Krise betroffen hier kaum Entsprechungen haben. Wo sie doch vorkommen, referieren sie oft gerade nicht auf sportliche, sondern auf wirtschaftliche Krisen: ${ }^{10}$

(8) Die Finanzkrise wird für den englischen Fußball-Spitzenclub Chelsea immer mehr zum Problem. Wie Chefscout und Nachwuchs-Direktor Frank Arnesen gegenüber der Zeitung "Guardian" zugab, wurde seinem schwer verschuldeten Klub von Eigentümer Roman Abramowitsch ein strikter Sparkurs verordnet, weil der russische Milliardär die Auswirkungen der Krise stark zu spüren bekommt. (Niederösterreichische Nachrichten, 23.12.08)

(9) Insgesamt steckt der italienische Fussball in finanziellen Nöten. [...] Ebenfalls von der Krise betroffen ist die AC Milan von Ex-Ministerpräsident Silvio Berlusconi. Sein Medienimperium Mediaset wird an der Börse abgestraft und die Werbeeinnahmen brechen ein. $(N Z Z, 03.08 .12)$

Eine solche Differenzierung zwischen der Krise einerseits und ihren Auswirkungen und Symptomen andererseits, die in korpuslinguistischen Forschungen als typisches Muster in Wirtschaftskrisendiskursen herausgestellt wurde (vgl. etwa Storjohann / Schröter 2013: 195; Ziem / Scholz / Römer 2013: 337, 353), ist bei Krisen im Fußball kaum möglich. Auch die für Krisendiskurse typischen Transitivkonstruktionen mit Krise als Satzsubjekt wie etwa Die Krise trifft $\mathrm{x}$ (vgl. Ziem / Scholz / Römer 2013: 351) sind nur selten, und dann meist im Kontext von wirtschaftlichen Krisen belegt:

10. Die Signifikanz des Attributs sportliche im Fußballkorpus zeigt bereits, dass eine nähere Charakterisierung und somit auch Abgrenzung gegenüber anderen Formen von Krisen von den Textproduzenten für nötig erachtet wird. 
(10) Krise trifft den Fußball. Die Wirtschaftskrise schlägt nun auch auf den italienischen Profifußball durch. (Hannoversche Allgemeine, 17.04.09)

Sportliche Krisen im Fußball bestehen demgegenüber schlicht in fehlenden sportlichen Erfolgen und sind genau dann schlagartig beendet, wenn sich die Erfolge wieder einstellen. Aufschlussreich ist in diesem Zusammenhang neben dem diminutiven Verb kriseln auch das Kompositum Ergebniskrise, das die Charakteristik von Krisen im Fußball überaus treffend einfängt:

(11) Wir haben beim 1:2 gegen Schalke gut gespielt, befinden uns aber in einer Ergebniskrise. (Nürnberger Zeitung, 20.02.14)

Das Spielergebnis, das die tatsächliche Spielqualität angeblich nicht widerspiegelt, ist Ursache, Wesen und Symptom der Krise zugleich. Die Frage nach Gründen wie auch nach möglichen Folgen der Krise erübrigt sich.

Wie eingangs bereits erwähnt, wird die Schnelllebigkeit von Krisen im Fußball oft auch von den Akteuren des Fußballdiskurses selbst thematisiert. Dass metasprachliche Bezugnahmen auf das Wort Krise häufig sind, lässt sich bereits an den signifikanten Kookkurrenzpartnern Wort, sprechen und einreden ersehen. Einige typische Belege seien hier genannt:

(12) Beim deutschen Fußball-Meister VfB Stuttgart, nächster Bundesliga-Gegner von Hannover 96, machte nach der 1:2-Pleite im Bundesliga-Duell beim Aufsteiger Hansa Rostock das Wort Krise wieder die Runde. (Rhein-Zeitung, 01.10.07)

(13) Einen katastrophalen Start in Rückrunde erwischte Sandkamp. Aus den letzten beiden Partien holten sich Kicker gleich neun Gegentore ab. Doch von einer Krise möchte SV-Coach Thomas Orth nicht sprechen. (Braunschweiger Zeitung, 01.04.10)

(14) „Wir haben wieder 90 Minuten guten Fußball gespielt und gezeigt, dass wir in keiner Krise stecken, wie man uns einreden wollte", erklärte Außenverteidiger Marcel Schmelzer. (Nürnberger Nachrichten, 21.02.11)

Die Journalisten ebenso wie die Spieler und Trainer haben offenbar ein Gespür dafür, dass Krisen im Fußball buchstäblich herbeigeredet werden.

Dass Krisen immer auch sprachlich konstruiert werden müssen, ist auch in der Forschung zu Wirtschaftskrisen gezeigt worden (vgl. Wengeler / Ziem 2013). Doch während allein schon die Diagnose von Wirtschaftskrisen mitunter aufwändige argumentative Verfahren der medial vermittelten Bewusstmachung verlangt (vgl. Wengeler 2013: 46-51), werden Krisen im Fußball womöglich schlicht mit Blick auf die Ergebnislisten ausgerufen, und gerade dies ist immer wieder ausdrücklich Thema. Bei der Durchsicht entsprechender Belege fällt dabei eine Tendenz auf: Metasprachliche Bezugnahmen werden meist an die Perspektive der Spieler und Trainer gebunden, und die mit diesen Bezugnahmen oft einhergehende Distanzierung bis hin zu einem regelrecht sprachkritischen Impetus wird von den Journalisten allenfalls referierend wiedergegeben. So wird ein Trainer wie folgt zitiert:

(15) „Überall hört man nur von Krisen. Ehekrise, Wirtschaftskrise, Finanzkrise, EHC-Krise - wenn einer will, kann er immer von einer Krise sprechen. Ich sage aber, wir haben eine Situation, in der wir derzeit nicht gewinnen." (Braunschweiger Zeitung, 17.12.2008) 
Auch eine im sprachkritischen Sinne inflationäre Verwendung des Ausdrucks Krise wird durchaus thematisiert, allerdings wird diese Sprachkritik an den betroffenen Verein delegiert:

(16) In München reagieren sie allergisch auf das Wort Krise, weil es inflationär benutzt wird, sobald Anspruch und Wirklichkeit etwas auseinanderklaffen. (Nürnberger Nachrichten, 24.02.12)

Gleichwohl ist zu bedenken, dass es die Journalisten selbst sind, die diese Kritik an ihren eigenen Krisenkonstruktionen präsentieren. Sie selbst sind es, die in ihren Texten die diskursive Praxis des Herbeiredens und des Abstreitens von Krisen inszenieren. In der im Korpus vierzig Mal belegten Formulierung Krise welche Krise? gerinnt diese Praxis zu einem stilisierten Dialog:

(17) Krise - welche Krise? Der FC Bayern ließ der Bundesliga-Trainingseinheit gegen Hoffenheim ein weiteres Schützenfest folgen. (Nürnberger Nachrichten, 14.03.12)

Die diskursive Konstruiertheit von Krisen im Fußball und ihre damit einhergehende Schnelllebigkeit wird in diesem Dialog geradezu performativ in Szene gesetzt.

\section{Kookkurrenzprofil von Katastrophe}

Besonders signifikante Kookkurrenzpartner von Katastrophe sind die folgenden Ausdrücke, wiederum nach absteigenden Signifikanzwerten sortiert: ${ }^{11}$ eine, war, einzige, wäre, mittlere, absolute, schlichtweg, reine, halbzeit, erste, sportliche, keine, totale, chancenauswertung, abstieg, reinste, schimpfte, zweite, größtmögliche, fukushima, nationale, chancenverwertung, hälfte, mittelschwere, ärgerte.

Bei der Durchsicht der Belege ergibt sich ein recht eindeutiges Bild. In $60 \%$ der Belege wird Katastrophe mit unbestimmtem Artikel (eine Katastrophe) gebraucht. In $43 \%$ der Belege handelt es sich um die Prädikativkonstruktionen ist / war / wäre / ... eine Katastrophe. In weiteren $15 \%$ der Belege wird eine Katastrophe mit einem zusätzlichen Attribut versehen, wobei es sich zumeist um Attribute handelt, die weniger die Art der Katastrophe (etwa humanitäre o.ä.) als vielmehr ihren Grad näher charakterisieren: eine einzige / mittlere / absolute / reine / totale Katastrophe. Es handelt sich bei dieser indefiniten Verwendung offenbar um eine verfestigte Wendung, die deshalb auch oft isoliert etwa als Überschrift Eine Katastrophe zu finden ist.

Das Bild komplettiert sich durch die typischen Subjekte: Insbesondere die erste und zweite Halbzeit / Hälfte sowie die Chancenauswertung können in diesem Sinne eine Katastrophe sein, wobei es sich meist um Urteile über soeben zu Ende gegangene Spiele handelt:

11. Hier liegen die Signifikanzwerte $\left(X^{2}\right)$ zwischen 163571 und 72 und somit ebenfalls über dem kritischen Wert von 10,83 für $p<0,001$. 
(18) „Die erste Hälfte war eine Katastrophe. Nach einem Donnerwetter war es in der zweiten Hälfte dann besser." (Rhein-Zeitung, 30.03.13)

(19) „Unsere Chancenauswertung war eine reine Katastrophe", ärgerte sich Leuchtmann trotz des Sieges, „wir hätten schon vor der Pause klar führen müssen." (Niederösterreichische Nachrichten, 29.08.13)

Für das Subjekt Abstieg ist die Kopula im Konjunktiv Präteritum typisch:

(20) Ein Abstieg wäre eine Katastrophe für den Verein, denn in der 3. Liga bist du erst einmal weg vom Fenster. (Hamburger Morgenpost, 03.03.15)

Es können hier offenbar zwei Verwendungsweisen unterschieden werden. Einerseits können bereits geschehene oder wie in Beispiel 20 auch nur drohende Ereignisse wie der Abstieg, eine Niederlage oder der Ausfall eines wichtigen Spielers als eine Katastrophe bezeichnet werden, um diese (durchaus im Rahmen der auch in Wörterbüchern verzeichneten Kernbedeutung dieses Ausdrucks) als folgenschweres Unglück zu charakterisieren. Andererseits können über konkrete Ereignisse hinausgehende Spielhandlungsweisen (Chancenauswertung, Passpiel, Leistung), aber auch Personen (Schiedsrichter) oder Objekte (Platz, Rasen, Ball) als eine Katastrophe bezeichnet werden. Der Aspekt der Folgenschwere tritt hier in den Hintergrund. Katastrophe dient hier in erster Linie dazu, etwas in drastischer Weise negativ zu bewerten (vgl. hierzu Schwarz-Friesel in diesem Band). In Anlehnung an elexiko wäre Katastrophe in dieser Verwendungsweise als Einstellungsprädikator ${ }^{12}$ zu beschreiben. Er kennzeichnet weniger einen Gegenstand als Exemplar einer Klasse und etabliert diesen als Referenten in der textlich vermittelten Sachverhaltsrepräsentation, sondern bringt (insbesondere in prädikativer Funktion) in erster Linie eine subjektive Einschätzung zum Ausdruck. In diesen Fällen wäre die Adjektivphrase sehr schlecht eine mögliche Paraphrase. ${ }^{13}$ Eine stichprobenhafte Auszählung von 100 zufällig ausgewählten Belegen mit ist / war / wäre eine Katastrophe zeigt dabei, dass in 70 Fällen eben diese Lesart als Einstellungsprädikator vorliegt. ${ }^{14}$

$\mathrm{Zu}$ diesem Befund passt, dass mit schimpfte und ärgerte (sich) zwei expressiv-emotive Redeverben (vgl. Harras et al. 2004: 324f.) signifikante Kookkurrenzpartner zu Katastrophe sind. Typische Beispiele sind die folgenden Belege:

12. Vgl. hierzu [http://www.owid.de/wb/elexiko/glossar/Einstellungspraedikatorhtml] Stand: 12.12 .2016 und die erläuternden Ausführungen in $\mathrm{Haß}$ (2005).

13. Dem Wörterbuch elexiko zufolge handelt es sich bei dieser Lesart um eine Metonymisierung der Lesart „folgenschweres Unglück“. Damit ist offenbar gemeint, dass die auf diese Weise als Katastrophe bezeichneten Handlungsweisen, Personen und Sachverhalte dazu führen, dass sich ein Unglück ereignet.

14. In einem Korpus von Fußball-Livetickern zu rund 5200 Partien von der Internetseite [www.weltfussball.de] (Meier 2017) ist denn auch eine ganze Reihe von Komposita mit Katastrophe als Erstglied nachweisbar, in denen eben die evaluierende Konstruktion $N$ ist eine Katastrophe in einem Wortbildungsprodukt gerinnt: Katastrophenpass, Katastrophenstart, Katastrophenfehler, Katastrophenabehr, Katastrophenleistung, Katastrophenbilanz usw. Komposita mit Katastrophe als Zweitglied sind demgegenüber kaum zu finden und bezeichnen dann meist Unglücksfälle wie Brandkatastrophe. 
(21) „Das war unterirdisch, ein Desaster! Die Abwehrarbeit war eine Katastrophe", schimpfte Haupt. Seine Mannschaft ließ alles vermissen, was für ein erfolgreiches Auftreten von Nöten gewesen wäre. (Braunschweiger Zeitung, 13.02.06)

(22) „Ein schlechtes Spiel. Eine Katastrophe“, ärgerte sich Eintracht-Trainer Dilsad Özsoy über den desolaten Auftritt seiner Elf vor der Pause. (Rhein-Zeitung, 02.05.12)

Die Bezeichnung eines Aspekts des meist verlorenen Spiels als Katastrophe wird oft als Ausdruck einer mitunter hochemotionalen negativen Bewertung (vgl. Schwarz-Friesel 2013: 185f.) präsentiert. Aber auch in den Fällen, in denen tatsächlich konkrete Ereignisse als Katastrophen bezeichnet und mithin als folgenschwere Unglücke beschrieben werden, handelt es sich zumeist um direkte Redewiedergaben. Sind also Krisen typischerweise Zuschreibungen von Journalisten, von denen sich die Spieler und Trainer distanzieren, verhält es sich bei Katastrophen gerade umgekehrt: Es handelt sich um Zuschreibungen der Spieler und Trainer selbst, die typischerweise der Formulierung von Kritik dienen. Journalisten übernehmen diese Kritik nur allzu gerne, machen diese aber als fremde Sicht kenntlich, um nicht selbst derart drastisch werten zu müssen (vgl. Hauser / Meier 2014: 134f.). Eine kritische metasprachliche Distanzierung, wie sie bei Krise häufig von den Spielern und Trainern formuliert wird, ist bei Katastrophe deshalb auch eher auf Seiten der Journalisten zu finden wie etwa in folgendem Kommentar:

(23) Katastrophe ist, wenn die Erde bebt, ein Meteorit ins Rathaus fliegt, ein Vulkan ausbricht. Was der HSV, immer noch Tabellenletzter der Bundesliga mit einem Punkt, im Moment durchmacht, ist eine Prüfung. (die tageszeitung, 19.09.11)

Aber auch jenseits der direkten Redewiedergaben machen Journalisten meist deutlich, dass die Kennzeichnung als Katastrophe eine an fremde Perspektiven gebundene Einschätzung ist. Vielfach wird denn auch expliziert, dass es sich um subjektive Bewertungen handelt, die der Textproduzent nur berichtend wiedergibt:

(24) Schneiders Fehlen wurde bei Bayer Leverkusen einstimmig als Katastrophe gewertet.

(Süddeutsche Zeitung, 06.12.07)

Auch das aus Wendungen wie gefühlte Ewigkeit geläufige Adjektiv gefühlt, das eine eigentlich objektive Größe als nur subjektiv zugänglich rahmt (vgl. Schröter i.E.) und vor allem seine emotionsauslösenden Eigenschaften fokussiert, kann mit Katastrophe kombiniert werden:

(25) Durch diese Ergebnisse ist unsere eigene Messlatte sehr hoch und die letzten drei Spiele ohne Sieg und Tor sind eine gefühlte Katastrophe. (Hamburger Morgenpost, 02.03.10)

Aufschlussreich sind auch die 190 Mal belegten Adverbialergänzungen mit für, die nicht nur das Betroffensein von der Katastrophe bezeichnen können wie in Katastrophe für den brasilianischen Fußball, sondern auch der Kennzeichnung von Subjektivität dienen:

(26) Gegen die Deutschen setzt es ein bitteres 1:6. Eine Katastrophe für Österreichs Sportreporter, die an Nausch kein gutes Haar lassen. (Die Presse, 07.06.08) 
Diesbezüglich ambig ist dagegen der folgende Beleg.

(27) Nicht nur für die Vereinsführung, auch für die Fans wären namenlose Gegner aus der Europa League eine Katastrophe. (die tageszeitung, 02.05.11)

Hintergrund des Artikels ist die vergleichsweise schlechte Platzierung des ansonsten erfolgsverwöhnten FC Bayern auf einem Tabellenplatz, der nur zum Start in der Europa League berechtigt, die gegenüber der Champions League der deutlich weniger attraktive und vor allem auch weniger rentable Wettbewerb ist. Die Einordnung der Europa League Teilnahme als Katastrophe, die aber für viele andere Vereine ein großer Erfolg wäre, wird vom Journalisten in geradezu ironisierender Weise als subjektive Einschätzung präsentiert, die er selbst sich nicht zu eigen machen möchte.

Gegenüber der Verwendung mit unbestimmtem Artikel sind definite Bezugnahmen deutlich unterrepräsentiert. Sieht man noch einmal von Formulierungen wie war die reinste Katastrophe $a b$, in denen der bestimmte Artikel vornehmlich emphatische Funktion hat, bleiben nur noch 400 Belege (8\%). Bei ihrer Durchsicht fällt auf, dass dann vornehmlich auf Katastrophen außerhalb von Fußballspielen referiert wird (etwa die Katastrophe von Fukushima, die sich schließlich auch im Kookkurrenzprofil niederschlägt), deren schwere Folgen jedoch auch den Fußballbetrieb in irgendeiner Weise betreffen:

(28) Die Teams aus Sendai, Kashima und Mito werden wegen der Folgen des Erdbebens und des anschliessenden Tsunamis am 11. März für ihre Heimspiele ausweichen. Die seit der Katastrophe ausgefallenen Partien sollen im Juli nachgeholt werden. (St. Galler Tagblatt, 24.03.11)

(29) Er erinnerte an den FC Liverpool, der nach seiner Verwicklung in die Katastrophe im Brüsseler Heysel-Stadion von 1985 mit 39 Toten für sieben Jahre aus den internationalen Wettkämpfen ausgeschlossen wurde. (Nürnberger Zeitung, 26.11.11)

Definite Bezugnahmen kennzeichnen den thematisierten Gegenstand als für den Rezipienten identifizierbar, sei es nun wegen Vorerwähnung im Text (Beispiel 28) oder auf der Grundlage von allgemeinem Weltwissen (Beispiel 29) (vgl. SchwarzFriesel / Consten 2014: 55). Die Seltenheit definiter Bezugnahmen mit die Katastrophe stützt somit zusätzlich die Beobachtung, dass die Verwendung des Lexems Katastrophe meist nicht dazu dient, einen Sachverhalt als Exemplar einer Klasse zu kennzeichnen, sondern sich oftmals im Bewertungsausdruck erschöpft. So sind generell Verwendungen jenseits von Prädikativa, etwa als Satzsubjekt (eine Katastrophe bahnt sich an, die Katastrophe nimmt ihren Lauf), als Attribut (Opfer / Ausmaß / Ursache der Katastrophe) oder als nominaler Kern von adverbial gebrauchten Präpositionalphrasen (nach der Katastrophe), Verwendungen also, in denen das Lexem im engen Sinne referierend gebraucht wird, erstaunlich selten. Auch anaphorische Wiederaufnahmen (30) oder Weiterführungen mit Relativsätzen (31) sind dementsprechend kaum zu finden und bezeichnen dann meist Katastrophen außerhalb des eigentlichen Fußballspiels:

(30) Die Saison 2011 im professionellen japanischen Fussball war gerade angepfiffen, als sie nach einer Woche durch das schwere Erdbeben, den Tsunami und die Havarie des 
Atomkraftwerks Fukushima Daiichi jäh unterbrochen wurde. Die Katastrophe absorbierte nicht nur alle Ressourcen des Landes, sie traf auch zwei Teams der 1. Division der J-League direkt. (NZZ, 20.03.12)

(31) Das mit Spannung erwartete Spiel war am Ende unwichtig, schwerste Krawalle vor Spielbeginn hatten zu 39 Toten unter den Zuschauern geführt. Eine Katastrophe, die die ganze Fußball-Welt in einen Schockzustand versetzte. (Nürnberger Zeitung, 29.05.15)

Das Gleiche gilt auch für die Verwendung als Komplexanapher diese Katastrophe, die komprimiert Evaluationen über einen zuvor eingeführten Referenten ausdrückt und in anderen Kontexten wie etwa der Terrorberichterstattung vielfach nachweisbar ist (vgl. Schwarz-Friesel 2013: 216f. und SchwarzFriesel / Consten 2014: 123ff.). Eines der wenigen Beispiele, das sich überhaupt finden lässt, ist dieses:

(32) Sollte Argentinien scheitern, würde dem Zauberfuß diese Katastrophe angelastet. (Mannheimer Morgen, 21.06.14)

Es handelt sich hier aber um Einzelfälle, die zudem meist ironisch distanziert zu sein scheinen, ${ }^{15}$ so wie auch Attribute wie nationale Katastrophe kaum als ernsthaft vertretene Einschätzungen des Journalisten verstanden werden dürften:

(33) Das Mutterland des Fußballs atmet auf, die nationale Katastrophe ist abgewendet. Die ,Three Lions' haben den Kopf aus der Schlinge gezogen und haben das Achtelfinale erreicht. (Hamburger Morgenpost, 24.06.10)

Katastrophe, so kann also festgehalten werden, dient im thematischen Bereich der Fußballberichterstattung also in erster Linie als Ausdruck negativer Evaluierung, die sich syntaktisch meist als Prädikativum mit unbestimmtem Artikel ( $\mathrm{x}$ ist / war eine Katastrophe) realisiert und als solche phraseologisch verfestigt ist. ${ }^{16}$ Durch verschiedene textpragmatische Mittel wird diese Evaluierung zumeist als subjektive Einschätzung zu erkennen gegeben, von denen sich die Journalisten oft deutlich distanzieren.

\section{Kontrastive Keywordanalysen}

Bisher wurden nur Texte aus der thematischen Domäne Fußball ausgewertet. Um aber einen Eindruck davon zu bekommen, ob es sich tatsächlich um fußballtypische Verwendungsweisen der Lexeme Krise und Katastrophe handelt, sollen die entsprechenden Belege aus den Fußballkorpora nun noch mit dem themenunspezifischen Referenzkorpus kontrastiert werden. Dazu habe ich aus

15. Vgl. hierzu die Theorie der Ironie von Sperber und Wilson (1992), die Ironie als Effekt des distanzierenden „echoic mention" beschreibt.

16. Es gibt eine ganze Reihe von insbesondere im alltagssprachlichen Register gebräuchlichen Mustern, die formseitig der Formulierung $x$ ist eine Katastrophe ähneln und ebenfalls vornehmlich evaluative Funktion haben: $x$ ist eine Bank / ein Unding / eine Wucht / der Hammer / die Hölle / der Wahnsinn / der Brüller. Allen Formulierungen ist gemein, dass das Prädikativum nicht referentiell gebraucht wird bzw. die spezifisch evaluative Funktion verloren geht, wenn es referentiell gebraucht wird. 
den oben (Teil 1) beschriebenen Suchen im DeReKo nur die Belegsätze aus dem Themenbereich Fußball exportiert und Keywordanalysen relativ zu den jeweiligen Belegsätzen aus dem Referenzkorpus durchgeführt.

Die Keywordanalyse der Korpora mit den Belegsätzen zu Krise zeigt, dass für das Fußballkorpus vor allem die Präpositionen aus und in hochsignifikant sind. ${ }^{17}$ Gegenüber den 35 \% der Belege, in denen Krise in den Nominalphrasen in / aus der/die Krise eingebettet sind, sind es im Referenzkorpus nur $15 \%$, von denen aber, das zeigt ein Blick in die Konkordanz, auch hier die meisten von Fußball handeln. Auch die raumbezogenen Ausdrücke tiefer, schritt, stürzt und schlittert erweisen sich in Kontrast zum Referenzkorpus als hochsignifikant. ${ }^{18}$ Die Beobachtung, dass im Fußballkontext gerade die Dynamik von Krisen thematisiert wird, wird hierdurch zusätzlich gestützt. In umgekehrter Suchrichtung erweist sich für das Referenzkorpus u.a. das Lexem Lösung als signifikant. Damit wird insbesondere auf politische Krisen (etwa die sogenannte Krim-Krise) Bezug genommen, für die in diplomatischen Anstrengungen auf internationaler Ebene Lösungen gesucht werden müssen. Diese Redeweise hat im Kontext des Fußballs keine Entsprechung.

Auch die Keywordanalyse der Korpora mit den Belegsätzen zu Katastrophe stützt die aus den Kookkurrenzprofilen gewonnenen Beobachtungen. Zunächst erweist sich der unbestimmte Artikel eine als hochsignifikant für das Fußballkorpus und führt die Keywordliste sogar an. ${ }^{19}$ Gegenüber den $60 \%$ der Belege mit eine Katastrophe sind es im Referenzkorpus gerade einmal $11 \%$. Den $15 \%$ der Belege mit einem eingefügten Attribut (eine mittlere Katastrophe) stehen im Referenzkorpus $6 \%$ gegenüber, wobei das häufigste Adjektiv hier humanitäre ist. Sind Formulierungen mit bestimmtem Artikel im Fußballkorpus mit $8 \%$ klar unterrepräsentiert, sind die im Referenzkorpus mit knapp $50 \%$ deutlich stärker vertreten. Auch die Kopula war, wäre und ist sowie die Personalpronomen wir und uns sind hochsignifikant; dies stützt die Beobachtung, dass Katastrophe vor allem der in Prädikativa formulierten kritischen Einschätzung, insbesondere der Selbsteinschätzung dient. In umgekehrter Suchrichtung erweisen sich u.a. die Lexeme Menschen, Leben und Opfer sowie Ausmaß als signifikant. Die in der Berichterstattung über Katastrophen im herkömmlichen Sinne übliche Angabe, wie vielen Menschen die Katastrophe das Leben gekostet habe, der üblichen Maßeinheit also für das Ausmaß einer Katastrophe, ist bei den Katastrophen im Fußball nicht relevant.

\section{Fazit}

Die hier eingenommene korpusanalytische Perspektive auf das Reden über Krisen und Katastrophen im Kontext der Fußballberichterstattung hat einige

\footnotetext{
17. $X^{2}=300,9$ (aus) und 64,2 (in).

18. $X^{2}>61,7$.

19. $X^{2}=672,5$.
} 
für diesen thematischen Bereich typische sprachliche Muster zutage gefördert, die Hinweise auf die jeweiligen Konzeptualisierungen in dieser Diskursdomäne geben. Krisen im Fußball sind typischerweise schnell vorübergehende Phasen mit schlechten Spielergebnissen und entsprechenden Tabellenpositionen, die den berichtenden Journalisten Anlass zu Kritik geben und mit dem Lexem Krise gebündelt und durch raummetaphorisch unterlegte Darstellungen auch bildhaftanschaulich beschreibbar werden. Es handelt sich dabei um eine Kategorie der Fremdzuschreibung, die von den betroffenen Akteuren häufig zurückgewiesen wird. Katastrophen im Fußball sind dagegen typischerweise Ereignisse oder auch Handlungsweisen, Personen und Objekte, die in drastischer Weise negativ bewertet werden. Der vornehmlich evaluativ-expressive Gehalt des Lexems Katastrophe wird meist in indefinit formulierten Prädikativa ist eine Katastrophe zum Ausdruck gebracht - eine verfestigte Konstruktion, die von definiten Konstruktionen deutlich zu unterscheiden ist. Es handelt sich hierbei zumeist um eine Kategorie der Selbstzuschreibung, um eine oft hochemotionale Form der Selbstkritik, die von den berichtenden Journalisten oft distanzierend (etwa durch direkte Redewiedergabe, Markierungen von Subjektivität oder ironisierende Beschreibungen) wiedergegeben wird.

Korpusanalytische Befunde über musterhafte Einbettungen der Lexeme Krise und Katastrophe können und müssen also pragmatisch angereichert werden. Rekurrente lexikalische Wahlen und syntagmatische Muster können als Niederschlag wiederkehrender Verwendungssituationen beschrieben werden (vgl. Müller 2012: 36). Dementsprechend sind die korpusanalytischen Befunde auf pragmatische Faktoren dieses Diskursbereichs zu beziehen. Hier sind z.B. die Rollen der Auskunft gebenden Spieler und Trainer einerseits und der kritisch berichtenden Journalisten andererseits zu erwähnen, aber auch die Vermittlungsarbeit, die Journalisten zwischen den hochemotionalen Äußerungen der Spieler und Trainer, der Anforderung an objektive Berichterstattung und dem Unterhaltungsbedürfnis der Lesenden leisten müssen. Aus dieser Diskurskonstellation beziehen die beschriebenen sprachlichen Muster ihre Funktionalität und werden erst hier verständlich.

DiezunächstnaheliegendeThese, dass das Redenüber Krisen und Katastrophen in der Fußballberichterstattung schlicht Ausdruck eines dramatisierenden Sprachgebrauchs ist, muss deshalb in zweierlei Hinsicht spezifiziert werden: Erstens machen sich je verschiedene Diskursakteure das Reden über Krisen und Katastrophen zu eigen und nutzen das evaluative Potenzial dieser Lexeme auf je besondere Weise als Formen der Fremd- oder aber Selbstkritik. Zweitens machen die Journalisten, die in ihren Texten diese Formen der Kritik textuell inszenieren, die diskursive Konstruiertheit von Krisen und Katastrophen durchaus deutlich. Dass das Ausrufen von Krisen und Katastrophen dramatisierend und vielleicht auch überdramatisierend ist, wird von den Journalisten keineswegs überspielt, sondern oftmals geradezu performativ in Szene gesetzt. Die Abgehobenheit fußballerischer Krisen und Katastrophen von dem, was üblicherweise unter diese Bezeichnungen fällt, wird immer wieder thematisiert. Und wenn in der Fußballberichterstattung doch einmal über politische oder wirtschaftliche Krisen 
oder Katastrophen im Sinne eines todbringenden Unglücks gesprochen wird, sind auch die sprachlichen Muster deutlich verschieden.

Nun kann der Fußball - insbesondere der medial vermittelte Fußball insgesamt als symbolhaftes Geschehen beschrieben werden, in das eine Gesellschaft das, was sie umtreibt, hineinprojizieren und im Modus des Spiels austragen kann. Dies gilt insbesondere für Unterschiede und Antagonismen zwischen sozialen Gruppen und das sich daraus ergebenden Einigungs-, aber auch Konfliktpotenzial (vgl. Gebauer 2016: 112-116). Fußball und die emotionale Anteilnahme am Fußball eignen sich in besonderem Maße zur kollektiven Identitätsstiftung, die von der Fußballberichterstattung maßgeblich mitgetragen wird. So sind etwa Lokal- und Nationalstereotype im medialen Fußball allgegenwärtig, aber hiervon getragene Agonalitäten werden auf das Spiel projiziert, wo sie gewissermaßen losgelöst vom Alltag ausgelebt werden können (vgl. Moser 2012).

Man könnte dieThese aufstellen, dass das Reden über Krisen und Katastrophen im Fußball eine ähnliche symbolische Funktion erfüllt. Vielleicht sind es gerade die,großen' Krisen in Politik und Wirtschaft, und der mühevolle und kontroverse gesellschaftliche Umgang mit ihnen, die in Fußballkrisen unter den Vorzeichen des Spielerischen reinszeniert werden können. Die Möglichkeit einer Bewältigung von "Krisen' durch simples Toreschießen mag hier ebenso kompensatorisch wirken wie die Möglichkeit, ,Katastrophen' wie den Ausfall eines wichtigen Spielers durch besonders gute Leistungen in ihren Folgen abzumildern. Eine solche These ist natürlich gewagt und müsste durch diachrone Analysen gestützt werden, für die jedoch keine Daten in ausreichender Menge vorliegen. Doch der korpusanalytische Blick auf typische sprachliche Muster des Redens über Krisen und Katastrophen im Bereich des Fußballs legt zumindest nahe, dass Inszeniertheit ein wichtiger Wesenszug dieser Krisen und Katastrophen ist, denen somit allenfalls ein spielerischer Ernst zukommt.

\section{Literaturverzeichnis}

\section{Korpora}

Abteilung Automatische Sprachverarbeitung, Universität Leipzig (2016) Wortschatz Universität Leipzig. [http://corpora.uni-leipzig.de/de], Stand: 12.12.2016.

Institut für Deutsche Sprache (2016) Deutsches Referenzkorpus / Archiv der Korpora geschriebener Gegenwartssprache 2016-I (Release vom 31.03.2016). Mannheim: Institut für Deutsche Sprache, [www.ids-mannheim.de/DeReKo], Stand: 12.12.2016.

Meier, Simon (Hrsg.) (2017) Korpora zur Fußballlinguistik (Release 2017-05). Fachgebiet Allgemeine Linguistik, Technische Universität Berlin, [www. fussballlinguistik.de/korpora], Stand: 23.06.2017. 


\section{Sekundärliteratur}

Anthony, Lawrence (2005) „AntConc: Design and Development of a Freeware Corpus Analysis Toolkit for the Technical Writing Classroom". In: IEEE International Professional Communication Conference Proceedings, [http://dx.doi.org/10.1109/IPCC.2005.1494244], Stand: 12.12.2016, S. 729-737.

Bondi, Marina (2010) „Perspectives on keywords and keyness. An introduction”. In: Marina Bondi / Mike Scott (Hrsg.), Keyness in texts, Amsterdam, Philadelphia: Benjamins, S. 1-18.

Bubenhofer, Noah (2009) Sprachgebrauchsmuster. Korpuslinguistik als Methode der Diskurs- und Kulturanalyse. Berlin, New York: de Gruyter.

Burkhardt, Armin (2006) "Sprache und Fußball. Linguistische Annäherung an ein Massenphänomen". Muttersprache 116, S. 53-73.

Gebauer, Gunter (2016) Das Leben in 90 Minuten. Eine Philosophie des Fußballs. München: Pantheon.

Harras, Gisela / Winkler, Edeltraud / Erb, Sabine / Proost, Kristel (2004) Handbuch deutscher Kommunikationsverben. Teil 1: Wörterbuch, Berlin, New York: de Gruyter.

Haß, Ulrike (2005) „Besonderheiten des Gebrauchs“. In: Ulrike Haß (Hrsg.), Grundfragen der elektronischen Lexikographie, Berlin, New York: de Gruyter, S. 265-276.

Hauser, Stefan (2010) „Der Live-Ticker in der Online-Berichterstattung: Zur Entstehung einer neuen Mediengattung". In: Hans-Jürgen Bucher / Thomas Gloning / Katrin Lehnen (Hrsg.), Neue Medien - neue Formate. Ausdifferenzierung und Konvergenz in der Medienkommunikation, Frankfurt a.M.: Campus, S. 207-225.

Hauser, Stefan / Meier, Simon (2014) „,Wer mich kennt, der weiß...' - Formelhafte Authentifizierung von Selbsteinschätzungen in Medieninterviews". Deutsche Sprache 42 / 2, S. 121-138.

Jung, Kerstin (2009) „Fußball als Medienereignis. Die mediale und sprachliche Inszenierung von Fußballevents als Sportspektakel“. In: Armin Burkhardt / Peter Schlobinski (Hrsg.), Flickflack, Foul und Tsukahara. Der Sport und seine Sprache, Mannheim, Leipzig, Wien, Zürich: Dudenverlag, S. 143-159.

Küster, Rainer (2010) „Im Tabellenkeller brennt noch Licht'. Metaphern in der Fußballsprache". Der Deutschunterricht 62 / 3, S. 26-37.

Lemnitzer, Lothar / Zinsmeister, Heike (2010) Korpuslinguistik. Eine Einführung. Tübingen: Narr.

Meier, Simon (2016) „Wahnsinnsdampfhammer". Fussballlinguistik, [http:// fussballlinguistik.de/2016/10/wahnsinn/], Stand: 01.03.2017. 
Moser, Fabrizio (2012) „Das Fußballritual als negativer Spiegel der Gesellschaft“. In: Christian Brandt / Fabian Hertel / Christian Stassek (Hrsg.), Gesellschaftsspiel Fußball. Eine sozialwissenschaftliche Annäherung, Wiesbaden: Springer VS, S. 67-84.

Müller, Marcus (2012) „Vom Wort zur Gesellschaft: Kontexte in Korpora. Ein Beitrag zur Methodologie der Korpuspragmatik". In: Ekkehard Felder / Marcus Müller / Friedemann Vogel (Hrsg.), Korpuspragmatik. Thematische Korpora als Basis diskurslinguistischer Analysen, Berlin, New York: de Gruyter, S. 33-82.

Ranerjee, Satanjeev / Pedersen, Ted (2003) „The Design, Implementation and Use of the Ngram Statistics Package". In: Alexander Gelbukh (Hrsg.), Computational Linguistics and Intelligent Text Processing. 4th International Conference, CICLing 2003 Mexico City, Mexico, February 16-22, 2003 Proceedings, Berlin, Heidelberg: Springer, S. 370-381.

Schröter, Juliane (i.E.) "Gefühlte Objektivität. Korpuspragmatischkulturanalytische Untersuchung eines neuen Phraseologismus". In: Bettina Bock / Konstanze Marx / Simon Meier / Robert Mroczynski / Sven Staffeldt (Hrsg.), Sprachliche Verfestigungen und sprachlich Verfestigtes, Linguistik Online, [https://bop.unibe.ch/linguistik-online/].

Schwarz, Monika (2002) „Konzeptuelle Ansätze II: Einebenen-Ansatz vs. Mehrebenen-Ansatz". In: D. Alan Cruse / Franz Hundsnurscher / Michael Job / Peter Rolf Lutzeier (Hrsg.), Lexikologie. Ein internationales Handbuch zur Natur und Struktur von Wortschätzen, Berlin, New York: de Gruyter, S. 277-284.

Schwarz-Friesel, Monika (2013) Sprache und Emotion. 2. Aufl., Tübingen, Basel: Francke.

Schwarz-Friesel, Monika / Consten, Manfred (2014) Einführung in die Textlinguistik. Darmstadt: Wissenschaftliche Buchgesellschaft.

Sperber, Dan / Wilson, Deirdre (1992) „On Verbal Irony“. Lingua 87, S. 53-76.

Steyer, Kathrin (2002) „Wenn der Hund mit dem Schwanz wedelt. Zum linguistischen Erklärungspotenzial der korpusbasierten Kookkurrenzanalyse". In: Ulrike Haß-Zumkehr / Werner Kallmeyer / Gisela Zifonun (Hrsg.), Ansichten der deutschen Sprache. Festschrift für Gehrhard Nickel zum 65. Geburtstag, Tübingen: Narr, S. 215-236.

Steyer, Kathrin (2004) „Kookkurrenz. Korpusmethodik, linguistisches Modell, lexikografische Perspektiven". In: Kathrin Steyer (Hrsg.), Wortverbindungen mehr oder weniger fest, Berlin, New York: de Gruyter, S. 87-116.

Storjohann, Petra / Schröter, Melani (2013) „Präsenz und Absenz lokaler Diskursgebrauchsmuster am Beispiel des deutschen und britischen Krisendiskurses". In: Martin Wengeler / Alexander Ziem (Hrsg.), Sprachliche Konstruktionen von Krisen. Interdisziplinäre Perspektiven auf ein fortwährend aktuelles Phänomen, Bremen: Hempen, S. 185-208. 
Weiß, Christian (2005) Online Publizierte Arbeiten zur Linguistik 1, Die thematische Erschließung von Sprachkorpora. [http://pub.ids-mannheim.de/ laufend/opal/pdf/opal2005-1.pdf], Stand: 12.12.2016.

Wengeler, Martin (2013) ", Unsere Zukunft und die unserer Kinder steht auf dem Spiel.' Zur Analyse bundesdeutscher Wirtschaftskrisen zwischen deskriptivem Anspruch und diskurskritischer Wirklichkeit". In: Ulrike Hanna Meinhof / Martin Reisigl / Ingo Warnke (Hrsg.), Diskurslinguistik im Spannungsfeld von Deskription und Kritik, Berlin: Akademie Verlag, S. 37-63.

Wengeler, Martin / Ziem, Alexander (2010) " ,Wirtschaftskrisen" im Wandel der Zeit. Eine diskurslinguistische Pilotstudie zum Wandel von Argumentationsmustern und Metapherngebrauch". In: Achim Landwehr (Hrsg.), Diskursiver Wandel, Wiesbaden: VS Verlag für Sozialwissenschaften, S. 335-354.

Wengeler, Martin / Ziem, Alexander (Hrsg.) (2013) Sprachliche Konstruktionen von Krisen. Interdisziplinäre Perspektiven auf ein fortwährend aktuelles Phänomen. Bremen: Hempen.

Ziem, Alexander / Scholz, Ronny / Römer, David (2013) „Korpuslinguistische Zugänge zum öffentlichen Sprachgebrauch. Spezifisches Vokabular, semantische Konstruktionen und syntaktische Muster in Diskursen über ,Krisen' ". In: Ekkehard Felder (Hrsg.), Faktizitätsherstellung in Diskursen, Berlin, New York: de Gruyter, S. 329-358. 
